\title{
Consideraçôes sobre a autonomia e a promoção da saúde
}

I ${ }^{1}$ Laura de Macedo Haeser, ${ }^{2}$ Fátima Büchele,

\author{
${ }^{3}$ Fabíola Stolf Brzozowski I
}

Resumo: Este artigo tem o objetivo de discutir a relação existente entre a autonomia individual e coletiva e a promoção da saúde. Assim, apresenta uma revisão bibliográfica sobre o percurso conceitual da promoção da saúde desde a perspectiva da Carta de Ottawa, destacando os momentos desse percurso onde é possível encontrar a ampliação da autonomia como um norte para as ações de promoção da saúde. Destaca a importância de as estratégias de promoção da saúde no Brasil priorizarem políticas públicas voltadas para a diminuição das iniquidades sociais, evidenciadas nas desigualdades em saúde, visando a ampliar a autonomia individual e coletiva.

> Palavras-chave: Promoção da saúde; autonomia; Carta de Ottawa; Saúde Pública.

\author{
1 Departamento Geral de \\ Hospitais do Ministério da \\ Saúde no Rio de Janeiro. \\ Endereço eletrônico: \\ jghduarte@hotmail.com \\ 2 Unidade de Pesquisa Clínica \\ (IFF/FIOCRUZ) Endereço \\ eletrônico: scgomes@iff. \\ fiocruz.br \\ ${ }^{3}$ Núcleo de Avaliação de \\ Tecnologias em Saúde (IFF / \\ FIOCRUZ). Endereço eletrônico: \\ mpinto@iff.fiocruz.br \\ ${ }^{4}$ Pós-Graduação em Saúde da \\ Criança e da Mulher, Núcleo de \\ Avaliação de Tecnologias em \\ Saúde (IFF / FIOCRUZ). Endereço \\ eletrônico: maria@iff.fiocruz.br
}

Recebido em: 22/06/2011. Aprovado em: 21/12/2011. 


\section{Introdução}

A promoção da saúde vem-se constituindo, desde a última década, como um campo importante de prática em saúde. Assim, ela se configura como uma estratégia fundamental da saúde coletiva no Brasil. Seu conceito vem se modificando e, hoje, está associado a um conjunto de valores - vida, saúde, solidariedade, equidade, democracia, cidadania, desenvolvimento, participação, dentre outros. Também se relaciona a uma combinação de estratégias visando a uma responsabilização mútua pela saúde, tais como ações do Estado (como as políticas públicas saudáveis), ações da comunidade (aumento da participação popular) e parcerias de vários setores (BUSS, 2009).

Ao observar a produção acadêmica relacionada ao tema da promoção da saúde, podem-se distinguir duas tendências de abordagens principais, conforme já descrito por Buss (2009) e Verdi e Caponi (2005): uma com enfoque comportamental, guiada pelos hábitos e estilos de vida (LOFREDO et al., 2001; CUNHA et al., 2009; SOARES; CEZAR-VAZ; SANT'ANA, 2011); e outra guiada pela concepção de qualidade de vida e pelos determinantes gerais das condiçōes de vida e saúde da sociedade (GUEDES; FERREIRA JUNIOR, 2010; SETTI; BOGUS, 2010; AZEVEDO; PELICIONI, 2011;).

A evolução do conceito de promoção da saúde se deve também à ampliação do conceito de saúde, hoje não mais considerada como ausência de doenças, mas composta pelos determinantes de condições sociais, de trabalho, ambientais e emocionais. Nesse sentido, encontra-se na promoção da saúde um forte incentivo a uma maior autonomia dos indivíduos e comunidades para que estes possam exercer uma independência sobre sua saúde.

Partindo da ideia de que a autonomia é tida como categoria central nas ações de promoção da saúde, este artigo tem como objetivo discutir a relação existente entre esses dois campos: promoção da saúde e autonomia, por meio de revisão de literatura. Para isso é abordado o conceito de promoção da saúde a partir da Carta de Ottawa, e autonomia, como sugerem Fleury-Teixeira et al. (2008), é entendida como a ampliação da capacidade de escolha das pessoas.

\section{A Promoção da Saúde}

Analisando o termo "promoção da saúde", logo o associamos com toda prática que se destina a promover saúde. No entanto, constitui-se como um campo próprio de 
práticas e ações específicas que se desenvolveram ao longo do tempo. Promoção é

um conceito conhecido desde a década de 1970, quando Leavell e Clark o definiram como um dos elementos do nível primário de atenção (CZERESNIA, 2009). Nos últimos 25 anos esse conceito, assim como sua prática, foi evoluindo, processo que se deu principalmente em países desenvolvidos como Canadá, Estados Unidos e na Europa ocidental (BUSS, 2009). A revalorização do conceito de promoção da saúde resgata, através de um discurso novo, o pensamento da medicina social do século XIX encontrado na obra de autores como Virchow e Villermée, para o qual a saúde depende das condições de vida (CZERESNIA, 2009).

Para se chegar ao conceito atual de promoção da saúde, aconteceram muitas discussões importantes, gerando documentos e concepções diversas sobre o tema. Basicamente podem-se distinguir, dentre os discursos em torno da promoção da saúde, duas grandes tendências, conforme já mencionado: uma comportamentalista e outra relacionada à qualidade de vida.

A primeira consiste na transformação dos comportamentos individuais, focando no estilo de vida e podendo projetar-se, no máximo, para a dimensão da família. Neste caso, as ações de promoção da saúde relacionam-se aos hábitos e estilos de vida, deixando de lado todos os fatores que estiverem fora do controle dos indivíduos. Como exemplo dessa abordagem, podemos citar a forma como algumas práticas encaram o aleitamento materno, o hábito de fumar, a dieta, a atividade física, etc. (BUSS, 2009). Assim, são priorizadas ações educativas relacionadas a fatores de risco comportamentais individuais, transferindo a responsabilidade pela saúde para o próprio indivíduo (VERDI; CAPONI, 2005).

A segunda grande tendência que caracteriza a moderna promoção da saúde é guiada pela concepção de qualidade de vida e direcionada pelos determinantes gerais das condiçõoes de vida e saúde da sociedade. Ou seja, saúde é o produto de uma série de fatores relacionados: alimentação, habitação, educação, trabalho, etc. Suas ações estariam mais voltadas ao coletivo de indivíduos e ao ambiente, por meio de políticas públicas focadas no desenvolvimento da saúde e na capacitação dos indivíduos e comunidades (BUSS, 2009; VERDI; CAPONI, 2005).

Neste trabalho, procuramos dar mais enfoque a alguns momentos principais dessa segunda tendência da promoção da saúde, hoje denominada como a "nova promoção da saúde", que são fundamentais para a discussão proposta acerca da autonomia. 
$\mathrm{Na}$ década de 1970, o ministro canadense Lalonde fez um estudo sobre os investimentos realizados pelos governos e seus resultados enquanto melhoria dos indicadores de saúde. Nesse estudo, investigou sobre a causalidade do processo saúde-doença no Canadá e constatou que a influência dos estilos de vida e do ambiente eram responsáveis por $80 \%$ das causas de doenças e que os investimentos em saúde não estavam atuando no controle dessas causas. Assim, pode questionar a atuação exclusiva da medicina nas práticas de saúde, responsabilizando o governo por outras medidas que ampliassem essas práticas, tais como o controle de fatores que influenciam o meio ambiente como a poluição do ar, a eliminação dos dejetos humanos, entre outros (WESTPHAL, 2008).

Em 1974, escreveu o Relatório Lalonde, propondo algumas dimensōes a serem consideradas para a elaboração das políticas de saúde: o ambiente, a biologia humana, os estilos de vida das pessoas e o sistema de saúde. Esse relatório tinha por objetivo "mostrar os links entre diferentes tipos de mortalidade e doença, por um lado, e suas causas subjacentes, por outro" (LALONDE, 1974, p. 9), sugerindo, assim, uma relação entre doença e condições sociais.

As discussões relacionadas à promoção da saúde não podem ser desvinculadas das discussões acerca do próprio conceito de saúde. Por essa razão, outro momento que trouxe também avanços nessa discussão foi a Conferência de Alma-Ata, que aconteceu na antiga União Soviética em 1978. Nessa conferência, a saúde foi entendida pela primeira vez como direito e como integrante de vários setores da sociedade e não apenas dos serviços de saúde, fator importante na discussão da intersetorialidade dentro da nova promoção de saúde. Quando começou a haver participação dos usuários nos serviços de saúde, as abordagens normativas, hierarquizadas e higienistas foram questionadas e, nesse cenário, a autonomia dos usuários passou a ser relacionada com a promoção da saúde (WESTPHAL, 2008). Segundo Buss (2009), a valorização do conhecimento popular e da participação social está na base conceitual da promoção da saúde.

$\mathrm{O}$ último marco a ser abordado aqui, que se configurou como principal documento conceitual para a concepção da promoção da saúde que se tem hoje, é a Carta de Ottawa, gerada na I Conferência Internacional sobre Promoção da Saúde, de 1986. Essa carta apresenta um conceito de promoção de saúde, e também de saúde, que extrapola as determinações biológicas focadas na doença:

Promoção da saúde é o nome dado ao processo de capacitação da comunidade para atuar na melhoria de sua qualidade de vida e saúde, incluindo uma maior participação no 
controle desse processo. Para atingir um estado de completo bem estar físico, mental e social os indivíduos e grupos devem saber identificar aspiraçōes, satisfazer necessidades e modificar favoravelmente o meio ambiente. A saúde deve ser vista como um recurso para a vida, e não como objetivo de viver. Nesse sentido, a saúde é um conceito positivo, que enfatiza os recursos sociais e pessoais, bem como as capacidades físicas. Assim, a promoção da saúde não é responsabilidade exclusiva do setor saúde, e vai para além de um estilo de vida saudável, na direção de um bem-estar global. (BRASIL, 2002, p.19).

Como vemos, o documento destaca a intersetorialidade, assim como aponta determinantes múltiplos para a saúde. Essa definição de promoção da saúde enfatiza a ação política e ambiental como fundamental para a melhoria da qualidade de vida e saúde, propondo a atuação da comunidade a partir da identificação das próprias necessidades. Nesse incentivo pela ação comunitária para a produção da saúde, ela é concebida, portanto, como recurso para a vida diária e não como meta a ser alcançada.

A Carta de Ottawa traz ainda alguns pré-requisitos para a saúde, ou seja, apresenta as condições e os recursos fundamentais para a saúde: paz, habitação, educação, alimentação, renda, ecossistema estável, recursos sustentáveis, justiça social e equidade (BRASIL, 2002). Além disso, propõe cinco campos de ação centrais para se promover saúde: a elaboração e implementação de políticas públicas saudáveis; a criação de ambientes favoráveis à saúde, o reforço da ação comunitária, o desenvolvimento de habilidade pessoais e a reorientação do sistema de saúde (BUSS, 2009). E especificamente sobre o reforço da ação comunitária e o desenvolvimento de habilidade pessoais, fala-se em empowerment comunitário e individual, respectivamente. Ou seja, fala-se de aquisição, por parte da comunidade e do indivíduo, de poder técnico e consistência política para atuar em prol de sua própria saúde (BUSS, 2009). Esse conceito está relacionado à ampliação da autonomia dos sujeitos, assunto que será abordado mais adiante.

No Brasil, em 2006, foi lançada a Política Nacional de Promoção da Saúde, com o objetivo de "Promover a qualidade de vida e reduzir vulnerabilidade e riscos à saúde relacionados aos seus determinantes e condicionantes - modos de viver, condiçõoes de trabalho, habitação, ambiente, educação, lazer, cultura, acesso a bens e serviços essenciais" (BRASIL, 2006, p.13). Com um dos focos na equidade em saúde, as ações de promoção da saúde, de acordo com a própria Política Nacional de Promoção à Saúde (BRASIL, 2006), objetivam reduzir as desigualdades em saúde e ampliar a autonomia e a corresponsabilidade das pessoas e dos coletivos (incluindo aqui o próprio poder público), no cuidado à saúde. 
Apesar de ser um avanço, o conceito ampliado de saúde e, consequentemente, o conceito de promoção da saúde, que leva em consideração as condiçôes de vida, as desigualdades e a vulnerabilidade social, traz um problema: ao se considerar a saúde em seu significado pleno, lida-se com "algo tão amplo como a própria noção de vida" (CZERESCNIA, 2009, p.50). Dessa forma, promover a vida em suas várias dimensões requer um esforço das ações do âmbito global de um Estado e também uma singularidade e autonomia dos sujeitos.

Os pré-requisitos e perspectivas para a saúde não são assegurados somente pelo setor saúde, por isso é preciso uma ação coordenada entre todos os envolvidos: governo, setor saúde, setores sociais e econômicos, organizações voluntárias, nãogovernamentais, autoridades locais, indústria e mídia. Além disso, como já foi dito, o conhecimento popular e a participação social estão na base da formulação conceitual da promoção da saúde. Assim, os grupos sociais têm papel importante na "mediação" entre os diferentes interesses, em relação à saúde, existentes na sociedade (BUSS, 2009, p.30). Dessa forma, podemos perceber que, a partir da Carta de Ottawa, a concepção de saúde utilizada nas práticas da promoção da saúde pode se dar através de uma perspectiva ampliada em relação àquela vinculada à ausência de doenças e centrada no comportamento dos indivíduos e seus estilos de vida, passando a considerar também os determinantes gerais, tais como condições sociais, ambientais e emocionais.

Segundo Westphal (2008), o entendimento ampliado de saúde, envolvendo os aspectos biológicos, sociais e econômicos, refere-se à relação existente entre os níveis de saúde da população e à maneira como a sociedade distribui seus recursos econômicos e sociais. Nessa lógica, Fleury-Teixeira et al. (2008, p.2.117) afirmam também que "as condições sociais são a base para o padrão sanitário do povo, assim como a posição de cada indivíduo na sociedade é um determinante fundamental da própria saúde". Ou seja, as condiçôes de vida, as relações sociais, o trabalho, o lazer, a educação, a qualidade de vida, são elementos determinantes para a constituição da saúde.

Fleury-Teixeira et al. (2008) referem que a percepção de que as condições sociais têm papel determinante nas condições de saúde é algo que se afirma desde a revolução industrial. E apontam, ainda, que

[...] o modo de ordenamento social e as posições recíprocas dos indivíduos na hierarquia social determinam largamente o modo como as pessoas se interacionam na 
sociedade, o que, por sua vez, determina as suas condições psíquicas, e isto constitui

um elo básico nas cadeias causais de todos os principais agravos e patologias da contemporaneidade (FLEURY-TEIXEIRA et al., p. 2.118).

Essas considerações acerca da influência das condições sociais para a saúde vêm ao encontro das discussões sobre a promoção da saúde que foram abordadas nesta revisão, ou seja, destacam a questão ambiental e política como potencialmente influentes na determinação da saúde. A promoção da saúde, ao respaldar-se nessas conviç̧óes, destaca a importância de as ações e práticas promoverem a ampliação do controle por parte dos indivíduos e dos coletivos sobre os determinantes de sua saúde; assim como a ampliação do poder de questionar, refletir, decidir e agir por parte dos mesmos. Pode-se perceber, a partir dessas reflexôes, uma relação da autonomia com a promoção da saúde, seus princípios e práticas (FLEURY-TEIXEIRA et al., 2008).

\section{A questão da autonomia na promoção da saúde}

De acordo com o que foi dito até aqui, a nova promoção da saúde permite identificar um norte para suas açôes voltadas ao incremento da saúde. Ou seja, garantir condiçôes dignas de vida e possibilitar que indivíduos e coletivos ampliem o controle ou o domínio sobre os determinantes de sua saúde (FLEURY-TEIXEIRA et al., 2008).

Retomando a Carta de Ottawa, o conceito de promoção da saúde está relacionado ao processo de capacitação da comunidade para atuar na melhoria de sua qualidade de vida e saúde, incluindo maior participação no controle desse processo (BRASIL, 2002). Dessa forma, os indivíduos e os grupos devem ser capazes de identificar e satisfazer suas necessidades. Traduzindo essa proposta, pode-se afirmar que quando as ações de promoção da saúde se propõem a ampliar o controle dos indivíduos sobre os determinantes da saúde, ela se refere também a ampliar a autonomia dos indivíduos e coletividades para agirem sobre esses determinantes. Encontra-se, portanto, a autonomia como categoria norteadora das ações em promoção da saúde (FLEURY-TEIXEIRA et al., 2008).

Essa abordagem de promoção da saúde também pode se voltar para o fortalecimento da ação comunitária, por meio do incremento do poder técnico e político das comunidades, também chamado de empowerment (VERDI; CAPONI, 2005). Segundo Carvalho (2004b), o empowerment suscita a 
elaboração de estratégias que procurem promover a participação visando ao aumento do controle sobre a vida por parte dos indivíduos e comunidades, a eficácia política, maior justiça social e melhoria da qualidade de vida. Ser/estar empowered significa ter autonomia para fazer escolhas informadas, objetivo primordial da promoção da saúde (OLIVEIRA, 2005).

Mas o que viria a ser autonomia? De acordo com o dicionário, encontramos a definição de autonomia como: faculdade de se ordenar por si mesmo; direito ou faculdade de se reger (uma nação) por leis próprias; liberdade ou independência moral ou intelectual; condição pela qual o homem pretende poder escolher pelas leis que regem sua conduta (FERREIRA, 1997). Autonomia está relacionada então à ideia de liberdade, de fazer suas próprias escolhas, da livre decisão dos indivíduos sobre suas próprias ações e da possibilidade de traçar suas trajetórias de vida (FLEURY-TEIXERIA et al., 2008). Refere-se à capacidade dos seres humanos de viverem suas vidas a partir de leis próprias. Supõe a condição de homem livre para assumir suas escolhas. A autonomia é o contrário da heteronomia, em que as leis são recebidas de outros (CAMPOS; CAMPOS, 2008).

A prescrição de homem livre, de liberdade de expressão, de apelo ao gozo e à felicidade pode ser considerada marca da sociedade capitalista. A aparente liberdade individual foi conquistada com a extensão das relações mercantis a todas as esferas da sociedade. Assim, na sociedade capitalista, o exercício de liberdade, muitas vezes, se dá através do consumo (FLEURY-TEIXEIRA et al., 2008; OLIVEIRA, 2005).

Autonomia seria uma característica das sociedades democráticas marcadas pelo direito a diversidade, livre expressão, liberdade de comportamentos de indivíduos e grupos, desde que respeitados os limites de danos a terceiros. Em uma democracia, a autonomia é relativa. A necessidade natural de socialização do homem faz com que ele se torne dependente das relaçôes sociais, fazendo com que sua possibilidade de autonomia seja sempre em relação aos outros ou ao coletivo. As regras postas para o processo civilizatório, como as leis, enquadram e limitam a autonomia de cada um. Numa sociedade, os diferentes grupos sociais gozam de diferentes graus de autonomia, o que demonstra a dimensão política da autonomia, uma vez que ela é uma consequência das relações de poder (CAMPO; CAMPOS, 2008; FLEURY-TEIXEIRA et al., 2008). 
Autonomia é relativa, pois viver em sociedade significa minimamente submeter-se a um conjunto de regras. Nesse sentido, como colocam Campos e Campos (2008), vale pensar a autonomia como "capacidade do sujeito de lidar com sua rede de dependência”. A constituição da autonomia depende do próprio sujeito e da coletividade. O sujeito é sempre responsável, junto com o coletivo, pela sua constituição e pela constituição do mundo que o cerca. Isso que dizer que a autonomia também depende de condiçôes externas ao sujeito, ainda que ele participe da criação dessas circunstâncias. Depende da organização de uma sociedade democrática e de suas leis; depende da educação, da saúde, da economia, da cultura, dos valores cultivados na sociedade em que ele está inserido, etc. Possibilitar maiores graus de autonomia depende, por exemplo, da oferta ao acesso à educação e à informação, como meio de formar cidadãos mais reflexivos e questionadores e assim mais potentes ao exercício de autonomia de “interferir na sua rede de dependência” (CAMPOS; CAMPOS, 2008).

Outra concepção que podemos ter de autonomia é aquela descrita por Menendez (2003, p.198), chamada de autoatenção e relacionada, especificamente, à área da saúde. Segundo esse autor, por autoatenção entende-se:

\footnotetext{
[...] as representações e práticas que a população utiliza ao nível de sujeito e grupo social para diagnosticar, explicar, atender, controlar, aliviar, suportar, curar, solucionar ou prevenir os processos que afetam sua saúde em termos reais ou imaginários, sem a intervenção central, direta e intencional de curadores profissionais, ainda quando estes podem ser a referência da atividade de autoatenção; de tal maneira que a autoatenção implica decidir a autoprescrição e o uso de um tratamento de forma autônoma ou relativamente autônoma.
}

De acordo com essa percepção, a autoatenção seria uma forma de autonomia inerente a todos os seres humanos, ou seja, todos possuem algum grau de autonomia, fato que algumas ações ditas de promoção da saúde muitas vezes ignoram, principalmente às de cunho comportamentalista. Nesse sentido, Menendez (2003) distingue autoatenção do conceito de autocuidado, este sim desenvolvido pela biomedicina principalmente a partir do conceito de estilo de vida. Para ele, o autocuidado são as ações desenvolvidas pelos indivíduos para prevenir o desenvolvimento de doenças e favorecer certos aspectos de saúde; refere-se a práticas individualistas e faz parte da autoatenção.

Levando em consideração os aspectos levantados até aqui e retomando a Carta de Ottawa (1986) e seus campos privilegiados de ação, encontramos dois que se 
referem mais diretamente à ampliação da autonomia, que são: o fortalecimento da ação comunitária e o desenvolvimento de habilidades pessoais.

O fortalecimento da ação comunitária pode se dar por meio do aumento do poder técnico e político das comunidades (empowerment), como já assinalado anteriormente, objetivando sua participação efetiva e concreta na tomada de decisões, escolha de prioridades e na elaboração de estratégias para alcançar um melhor nível de saúde. Assim, é importante garantir o acesso às informações e às oportunidades de aprendizagem sobre as questões de saúde, para que os indivíduos possam tomar suas próprias decisões (VERDI; CAPONI, 2005).

Com o foco na questão do desenvolvimento de habilidades e atitudes favoráveis à saúde, a promoção da saúde aponta estratégias para a informação e a educação em saúde, visando a aumentar as possibilidades de a população exercer maior controle sobre si mesma e sobre o ambiente, e optando por opçōes mais favoráveis à saúde (VERDI; CAPONI, 2005).

Voltando ao conceito de autoatenção, é necessário que os profissionais de saúde levem em consideração esse aspecto apontado por Menendez (2003), gerando uma relação horizontal e de diálogo. De outro modo, as próprias ações de promoção da saúde, como educação em saúde, por exemplo, podem se tornar paternalistas, ou seja, ações baseadas no autoritarismo. Essa prática acaba por limitar as liberdades individuais ao invés de ampliá-las, papel central da promoção da saúde.

\section{Considerações sobre o princípio da autonomia na promoção da saúde e suas implicações éticas}

De acordo com o que vimos, a promoção da saúde possui um longo campo conceitual e de formulações práticas, que impõe implicações éticas. Estas podem ser estudadas com base em teorias e referências provenientes do campo da bioética. Abordaremos aqui, de forma sucinta, algumas reflexões sobre o tema da ampliação da autonomia na promoção da saúde à luz da bioética.

$\mathrm{O}$ respeito à autonomia do outro é um princípio ético fundamental. $\mathrm{E}$ isso significa respeitar a possibilidade do outro agir como autônomo, como sujeito de sua própria vida. $\mathrm{O}$ respeito à autonomia está relacionado à compreensão do direito do outro em ter um modo de pensar, fazer escolhas e orientar suas ações de acordo com seus valores e crenças (LIMA, 2008). No entanto, pode ser difícil pensar 
em autonomia nesse sentido, de respeito ao modo de pensar do outro, quando se tenta impor um estilo de vida ou comportamento a esse outro. A autonomia na promoção da saúde não pode ser pensada de forma vertical, em que o profissional da saúde informa o paciente qual é a melhor forma de agir para garantir sua saúde, para que o paciente possa, enfim, decidir, sobre sua saúde (dentro dessa visão, o paciente, agora informado, irá autonomamente decidir por acatar as sugestôes do profissional). Respeitar autonomia e contribuir para a autonomia, na promoção da saúde, não estão relacionados somente com o conhecimento técnico sobre saúde. Englobam também um aspecto político e social de reforço da cidadania e da criticidade, e que, por isso, não está restrito apenas à área da saúde.

Verdi e Caponi (2005) alertam para o risco de as políticas públicas de saúde serem construídas de forma reducionista e assim transformar problemas sanitários complexos em desvios de condutas individuais, transferindo a responsabilidade do Estado em produzir respostas efetivas ao próprio indivíduo, característica do enfoque comportamentalista da promoção da saúde. Nessa lógica, são formulados alguns programas de promoção da saúde focados na promoção de uma conduta individual sadia (VERDI; CAPONI, 2005).

Assim, as ações de promoção da saúde são desenvolvidas basicamente por meio de estratégias que promovem o desenvolvimento de hábitos e estilos de vida saudáveis, o que provoca implicações éticas. Ou seja, essas estratégias focalizam suas açóes no comportamento individual considerado de risco e acabam por produzir uma dupla responsabilização do sujeito. Primeiro porque ele passa a ser responsável por seu adoecimento, devido a seu comportamento de risco e, segundo, porque passa a ser responsabilizado pelo desenvolvimento de condutas e hábitos que evitarão o risco (VERDI; CAPONI, 2005). É nesse sentido que Carvalho (2004a) afirma que muitos projetos de promoção da saúde têm dificuldade de superar a tendência behaviorista (comportamentalista), mantendo o foco em ações voltadas para a regulação da vida social e estabelecimento de critérios que delimitam o sentido do viver saudável.

Em consonância com o exposto acima, percebe-se que através do desenvolvimento de hábitos saudáveis, as estratégias para promover saúde podem se tornar estratégias de vigilância que muitas vezes limitam e restringem as liberdades e decisões individuais (CARVALHO, 2004a; VERDI; CAPONI, 2005). Neste ponto é possível encontrar um entrave para a promoção da autonomia, porque a 
tendência de responsabilização dos indivíduos, caso estes não adotem condutas e hábitos saudáveis de vida, gera culpabilização e "subalternidade" às medidas médicas, sem possibilidade de construir autonomia (VERDI; CAPONI, 2005).

Carvalho (2004a), reforçando essa ideia, afirma que a autonomia proposta pela nova promoção da saúde estaria a priori formatada pelas informações preconcebidas de profissionais ou pelas diretrizes das Políticas Públicas Saudáveis. Assim, de acordo com o que viemos desenvolvendo, a autonomia é considerada categoria norteadora das ações em promoção da saúde voltadas ao fortalecimento da ação comunitária. No entanto, por meio das estratégias de desenvolvimento de hábitos, atitudes e estilos de vida favoráveis à saúde, reiterase o caráter de vigilância de comportamentos e, desta forma, a possibilidade de autonomia pode estar sendo enfraquecida (VERDI; CAPONI, 2005), além de ignorar o fato de que as pessoas possuem naturalmente certo grau de autonomia. Nesse sentindo, há um risco real de expropriação da saúde devido à tendência de delegar ao profissional de saúde a definição dos problemas de saúde, assim como as estratégias e as decisões a seguir, como se tratassem de questôes somente técnicas (CARVALHO, 2004a; VERDI; CAPONI, 2005).

Por fim, as estratégias de promoção da saúde, como estão sendo construídas, não se configuram como campo de exercício de participação e escolhas das pessoas, pois é possível perceber, nas práticas de promoção da saúde, um caráter paternalista nas relações entre profissional e usuário, o que impede esse usuário de se tornar um sujeito possuidor de direitos e com poder de decisão sobre sua saúde.

\section{Considerações finais}

O conceito de promoção de saúde e suas estratégias de ação evoluíram junto com a mudança na concepção de saúde. Assim, ao passo que a saúde deixou de ser considerada como ausência de doenças e passou englobar, além dos aspectos biológicos, os aspectos sociais e econômicos, mudou-se a forma de se pensar a promoção da saúde. Nessa lógica, encontram-se duas formas de abordá-la, a primeira focada na modificação dos comportamentos individuais e a segunda, caracterizando a moderna promoção da saúde, direcionada pelos determinantes gerais das condições de vida e saúde da sociedade, com ações voltadas ao coletivo de indivíduos e ao ambiente, por meio de políticas públicas focadas no desenvolvimento da saúde e na capacitação dos indivíduos e comunidades. 
Conforme essa segunda abordagem, a promoção da saúde pretende, por meio de suas ações, ampliar o controle por parte dos indivíduos e coletivos sobre os determinantes de sua saúde, ampliar o poder de questionar, refletir, decidir e agir por parte dos mesmos; ou seja, pretende apoiar os indivíduos e comunidades, possibilitando escolhas conscientes de acordo com suas necessidades e levando em consideração os saberes populares e a autoatenção. Nesse sentido, se pode identificar a ampliação da autonomia como um norte para as ações de promoção da saúde.

No entanto, vimos que as ações de promoção da saúde baseadas no desenvolvimento de hábitos e estilos de vida saudável podem enfraquecer a possibilidade de autonomia individual ou coletiva, pois apresentam dificuldades para superar a tendência comportamentalista, mantendo o foco em ações voltadas para a regulação da vida social e estabelecimento de critérios que delimitam o sentido do viver saudável. Assim, essas açôes para promover saúde podem se tornar estratégias de vigilância que muitas vezes restringem as liberdades e decisões individuais.

Como podemos perceber, é importante refletir sobre as implicações das açõos dos trabalhadores de saúde. É preciso também considerar a sociedade como um espaço de disputa de interesses políticos, individuais e coletivos, que envolvem forças desiguais, valores diferentes, crenças divergentes e que tudo isso está em jogo nesse processo de construção da promoção da saúde (VERDI; CAPONI, 2005). Nesse sentido, é importante que a promoção da saúde brasileira possa pensar em políticas públicas voltadas para a diminuição das iniquidades sociais, evidenciadas nas desigualdades em saúde. Pensar em políticas públicas saudáveis com foco na eliminação das múltiplas carências cotidianas da vida individual e coletiva para então alcançar o fortalecimento da ação comunitária necessária ao exercício da autonomia. ${ }^{1}$

\section{Referências}

AZEVEDO, E.; PELICIONI, M.C.F. Promoção da saúde, sustentabilidade e agroecologia: uma discussão intersetorial. Saúde e Sociedade, v.20, n.3, p.715-29, 2011.

BRASIL. Ministério da Saúde. Secretaria de Vigilância em Saúde. Política nacional de promoção da saúde. Brasília: Ministério da Saúde, 2006. Disponível em: http://portal.saude. gov.br/portal/arquivos/pdf/pactovolume7.pdf. Acesso em: $07 \mathrm{dez} 2010$. 

Secretaria de Políticas de Saúde. Projeto Promoção da Saúde. As Cartas da Promoção da Saúde. Brasília: Ministério da Saúde, 2002. Disponível em: http://bvsms.saude.gov.br/ bvs/publicacoes/cartas_promocao.pdf. Acesso em: 13 jun 2010.

BUSS, P.M. Uma introdução ao Conceito de Promoção da Saúde. In: CZERESNIA, D.; FREITAS, C.M.de (org.). Promoção da Saúde: conceitos, reflexões, tendências. Rio de Janeiro: Fiocruz, 2009. p.19-42.

CAMPOS, G.W.S. Co-construção de autonomia: o sujeito em questão. In: CAMPOS, G.W.S. et al. Tratado de Saúde Coletiva. São Paulo: Hucitec, 2008. p.669-688.

CARVALHO, S.R. As contradições da promoção à saúde em relação à produção de sujeitos e a mudança social. Ciência \& Saúde Coletiva, v.9, n.3, p.669-78, 2004 a.

. Os múltiplos sentidos da categoria "empowerment" no projeto de Promoção à Saúde. Cadernos de Saúde Pública, v.20, n.4, p.1-088-95, 2004 b.

CUNHA, R.R. et al. Promoção da saúde no contexto Paroara: possibilidade de cuidado de enfermagem. Texto Contexto \& Enfermagem, v.18, n.1, p.170-6, 2009.

CZERESNIA, D. O conceito de saúde e a diferença entre prevenção e promoção. In: CZERESNIA, D.; FREITAS, C.M.de (org.). Promoção da saúde: conceitos, reflexões, tendências. 2. ed. rev. e amp. Rio de Janeiro: Fiocruz, 2009.

FERREIRA, A.B.H. Dicionário Aurélio da lingua portuguesa. Rio de Janeiro: Nova Fronteira, 1997.

FLEURY-TEIXEIRA, P. et al. Autonomia como categoria central no conceito de promoção de saúde. Ciência \& Saúde Coletiva, v.13, sup. 2, p.2115-22, 2008.

GUEDES, L.M.; FERREIRA JUNIOR, M. Relaçôes disciplinares em um centro de ensino e pesquisa em práticas de promoção da saúde e prevenção de doenças. Saúde e Sociedade, v.19, n.2, p.260-72, 2010.

LALONDE, M. A new perspective on the health of Canadians: a working document. Ottawa: Government of Canada, 1974.

LIMA, R.G.S. Concepçôes de médicos de família no Brasil e na Itália sobre autonomia e solidariedade: implicações éticas para o cuidado na Atenção Primária de Saúde. 2008. 175 f. Mestrado (Dissertação de Mestrado) - Programa de Pós-Graduação em Saúde Pública, Universidade Federal de Santa Catarina, Florianópolis, 2008.

LOFREDO, S.M. et al. Investigação e controle de epidemia de escabiose: uma experiencia educativa em aldeia indígena. Saúde e Sociedade, v.10, n.1, p.65-86, 2001.

MENENDEZ, E. Modelos de atención de los padecimientos: de exclusiones teóricas y articulaciones prácticas. Ciência \& Saúde Coletiva, v.8, n.1, p.185-207, 2003.

OLIVEIRA, D.L. A 'nova' saúde pública e a promoção da saúde via educação: entre a tradição e a inovação. Revista Latino-Americana de Enfermagem, v.13, n.3, p.423-31, 2005. 
SETTI, A.F.F.; BOGUS, C.M. Participação comunitária em um programa de intervenção em área de proteção ambiental. Saúde e Sociedade, v.19, n.4, p.946-60, 2010.

SOARES, J.F.S.; CEZAR-VAZ, M.R.; SANT'ANNA, C.F. Prevenção de agravos e promoção da saúde: um estudo com trabalhadores portuários. Texto \& Contexto Enfermagem, v.20, n.3, p.225-34, 2011.

VERDI, M.; CAPONI, S. Reflexões sobre a promoção da saúde numa perspectiva da bioética. Texto \& Contexto Enfermagem, v.14, n.1, p.82-8, 2005.

WESPHAL, M.F. Promoção da Saúde e Prevenção de Doenças. In: CAMPOS, G.W.S. et al. Tratado de Saúde Coletiva. São Paulo: Hucitec, 2008. p. 635-668.

\section{Nota}

${ }^{1}$ L.M. Haeser e F. Büchele participaram de todas as etapas de elaboração do artigo; F.S.Brzozowski trouxe contribuições teóricas e realizou a revisão do artigo. 
Considerations on autonomy and health promotion

This paper aims to discuss the relation between individual and collective autonomy and health promotion. In order to do so, it reviews the literature on the conceptual course of health promotion since the Ottawa Charter, emphasizing the amplification of autonomy as a guide for health promotion actions. It also highlights the importance of health promotion strategies in Brazil, which prioritize public policies in order to decrease social inequalities. In particular, Brazilian policies are addressed towards increasing individual and collective autonomy.

> Key words: Health promotion; autonomy; Ottawa Charter, public health. 